\title{
Extracellular Histones Inhibit Fibrinolysis through Noncovalent and Covalent Interactions with Fibrin
}

\author{
Matthew Locke ${ }^{1} \quad$ Colin Longstaff ${ }^{1}$ \\ ${ }^{1}$ Biotherapeutics Division, National Institute for Biological Standards \\ and Control, South Mimms, Hertfordshire, United Kingdom \\ Thromb Haemost 2021;121:464-476.
}

\author{
Address for correspondence Matthew Locke, DPhil, Biotherapeutics \\ Division, National Institute for Biological Standards and Control, \\ South Mimms, Hertfordshire EN6 3QG, United Kingdom \\ (e-mail: Matthew.locke@nibsc.org).
}

\begin{abstract}
Keywords

- fibrin(ogen)

- fibrinolysis

- histones

- neutrophil extracellular traps

- FXIII

Histones released into circulation as neutrophil extracellular traps are causally implicated in the pathogenesis of arterial, venous, and microvascular thrombosis by promoting coagulation and enhancing clot stability. Histones induce structural changes in fibrin rendering it stronger and resistant to fibrinolysis. The current study extends these observations by defining the antifibrinolytic mechanisms of histones in purified, plasma, and whole blood systems. Although histones stimulated plasminogen activation in solution, they inhibited plasmin as competitive substrates. Protection of fibrin from plasmin digestion is enhanced by covalent incorporation of histones into fibrin, catalyzed by activated transglutaminase, coagulation factor FXIII (FXIIla). All histone subtypes ( $\mathrm{H} 1, \mathrm{H} 2 \mathrm{~A}, \mathrm{H} 2 \mathrm{~B}, \mathrm{H} 3$, and $\mathrm{H} 4)$ were crosslinked to fibrin. A distinct, noncovalent mechanism explains histone-accelerated lateral aggregation of fibrin protofibrils, resulting in thicker fibers with higher mass-to-length ratios and in turn hampered fibrinolysis. However, histones were less effective at delaying fibrinolysis in the absence of FXIIla activity. Therapeutic doses of low-molecular-weight heparin (LMWH) prevented covalent but not noncovalent histone-fibrin interactions and neutralized the effects of histones on fibrinolysis. This suggests an additional antithrombotic mechanism for LMWH beyond anticoagulation. In conclusion, for the first time we report that histones are crosslinked to fibrin by FXIIla and promote fibrinolytic resistance which can be overcome by FXIIla inhibitors and histone-binding heparinoids. These findings provide a rationale for targeting the FXIII-histone-fibrin axis to destabilize fibrin and prevent potentially thrombotic fibrin networks.
\end{abstract}

\section{Introduction}

Histones are released from damaged, dying, or activated cells during infection, inflammation, and trauma. ${ }^{1}$ A major source of extracellular histones are neutrophils, which eject webs of decondensed chromatin as neutrophil extracellular traps (NETs). ${ }^{2}$ NETs trap and kill microbial pathogens, either directly or by acting as a scaffold for the localized activation of coagulation, a process termed "immunothrombosis."3 However, NETs have been described as a double-edged sword, as their over- production or inadequate clearance can be harmful to the host and contribute to pathological microvascular thrombosis in sepsis and disseminated intravascular coagulation. ${ }^{4}$ NETs are also implicated in the development of large vessel occlusions, ${ }^{5}$ and their presence in circulation is associated with poor prognosis in cardiovascular and cerebrovascular diseases. ${ }^{6}$ The mechanisms by which NETs promote thrombosis are unclear but are likely to be multifactorial and involve recruitment and activation of soluble clotting factors, platelets, and leukocytes. ${ }^{7}$ NETs have been identified in patient thrombi ${ }^{8}$ and received

April 25, 2020

accepted after revision

September 15, 2020

published online

November 1, 2020 (c) 2020. Thieme. All rights reserved. Georg Thieme Verlag KG,

Rüdigerstraße 14,

70469 Stuttgart, Germany
DOI https://doi.org/

10.1055/s-0040-1718760. ISSN 0340-6245. 
intercalate into fibrin networks, making clots more stable and resistant to degradation. ${ }^{5}$ As the major protein component of NETs, histones are causally implicated in the formation of arterial and venous thrombi. ${ }^{6}$ However, most studies have focused on the procoagulant activities of histones, with fewer studies examining their antifibrinolytic mechanisms.

Fibrinolysis requires two key steps: the generation of plasmin from the inactive zymogen plasminogen, catalyzed by tissue type-plasminogen activator (tPA), followed by the subsequent digestion of fibrin by plasmin. These events are influenced by the fibrin structure and network architecture, which is in turn determined by the conditions at the time of fibrin formation. ${ }^{9}$ In blood, fibrinogen circulates in an environment rich in macromolecules and noncovalently binds a multitude of plasma proteins and polymers which can influence fibrin polymerization, structure, and susceptibility to fibrinolysis. While it is generally accepted that thicker fibrin fibers lyse more quickly than thinner fibers on a macroscopic scale, ${ }^{10}$ the relationship between fibrin structure and fibrinolysis is complex and varies depending on the fibrin(ogen)interacting protein or polymer and their electrostatic charge. Negatively charged DNA promotes the formation of densely packed networks of thick fibers less susceptible to plasmin digestion, ${ }^{11}$ whereas polyphosphate, another anionic polymer, gives rise a heterogeneous clot structure and attenuated plasmin generation. ${ }^{12}$ It has been known for some time that histones, which are rich in positively charged lysine and arginine residues, interact with fibrin(ogen) and increase resistance to fibrinolysis. ${ }^{13-16}$ However, the exact mechanisms behind these effects have not been fully elucidated. Histones interfere with the lateral organization of protofibrils, resulting in a fibrin network that is more resistant to tPA-mediated fibrinolysis, despite being composed of thicker fibers. ${ }^{17}$ It has been suggested that histone-induced structural alterations in fibrin mediate lytic resistance, as histones neither inhibited the caseinolytic activity of plasmin nor acted as plasmin substrates. ${ }^{16}$ Protamine, another strongly cationic protein, also induces thicker fibrin fibers, but with increased susceptibility to tPA lysis. ${ }^{18}$ These observations suggest that structural alterations in fibrin do not fully explain the antifibrinolytic effects of histones.

Another important determinant of the mechanical and biochemical stability of fibrin is factor XIII (FXIII) which is bound to fibrinogen in circulation. ${ }^{19}$ FXIII is activated by thrombin in the presence of calcium ions to produce the active transglutaminase, FXIIIa. ${ }^{19}$ FXIIIa introduces covalent isopeptide bonds (or crosslinks) between glutamine and lysine residues in the $\alpha$ and $\gamma$ chains of fibrin monomers within individual fibers. This creates $\gamma-\gamma$ dimers and high molecular weight $\alpha-\alpha$ and $\gamma-\alpha$ polymers, which increases clot stiffness and mediates red blood cell retention in clots. ${ }^{20}$ FXIIIa also covalently crosslinks other plasma proteins to fibrin, including antifibrinolytic molecules such as $\alpha_{2}$-antiplasmin, plasminogen activator inhibitor 2 (PAI-2), and thrombin activatable fibrinolysis inhibitor (TAFI). ${ }^{19}$ This allows FXIIIa to inhibit fibrinolysis, which is particularly apparent under flow ${ }^{21}$ and clot contraction conditions. ${ }^{22}$ Together, these activities make FXIIIa a critical determinant of fibrin network stability and thrombus composition and size, and a target in thrombotic disease. ${ }^{23}$

We previously found that histones colocalize with fibrin in patient thrombi and make clots more resistant to fibrinolysis. ${ }^{17,24}$ In the current study, we investigated the mechanisms behind these observations and show that histones competitively inhibit plasmin to delay fibrinolysis. Furthermore, the antifibrinolytic effects of histones are enhanced by covalent crosslinking to fibrin, catalyzed by FXIIIa. Our results suggest blocking histone-fibrin crosslinking with FXIIIa inhibitors or histone-binding heparinoids is an effective strategy to destabilize clots containing histones. These findings are relevant for the prevention of thrombotic disease associated with histone release.

\section{Methods}

\section{Reagents}

FXIII (code 02/170), tPA (code 98/714), and freeze-dried control plasma (code 06/158) were from NIBSC (South Mimms, United Kingdom). Thrombin was from Diagnostic Reagents Ltd. (Thame, United Kingdom). Antifibrinogen antibodies (A0080) were from Dako (Glostrup, Denmark). Anti-histone H3 (ab1791) and anticitrullinated histone H3 (ab5103) were from Abcam (Cambridge, United Kingdom). Anti-his tag antibodies (A00186) were from Genscript (New Jersey, United States). Mixed histones (calf thymus, IIA) and recombinant peptidyl arginine deiminase 4 (PAD4) were from Sigma (Poole, United Kingdom). Individual recombinant human histones expressed with an $\mathrm{N}$-terminal $\mathrm{His}_{6}$-tag were from Amsbio (Oxford, United Kingdom). Human fibrinogen (plasminogendepleted) was from Merck (Watford, United Kingdom). FXIIIdepleted plasma and glu-plasminogen were from Hyphen Biomed (Neuville-sur-Oise, France). T101 was from Zedira (Darmstadt, Germany) and prepared in HEPES-buffered saline (HBS; $10 \mathrm{mM}$ HEPES pH 7.4, $150 \mathrm{mM} \mathrm{NaCl}$ ). Chromogenic substrates for plasmin (S-2251) and tPA (S-2288) were from Chromogenix (Milan, Italy). Low-molecular-weight heparin (LMWH; Dalteparin/Fragmin) was from Pfizer (Kent, United Kingdom).

\section{Solution Chromogenic Assays}

For studying plasmin/tPA inhibition by histones, rates of hydrolysis of chromogenic substrates (S-2251 or S-2288 for plasmin and tPA, respectively) were obtained over a range of substrate and histone concentrations using online apps. ${ }^{25}$ Kinetic parameters were obtained by fitting plots of plasmin velocity as a function of S-2251 concentration to MichaelisMenten kinetics in GraphPad Prism (GraphPad software version 8.1.1, La Jolla, California, United States). Data were also plotted as double-reciprocal (Lineweaver-Burk) plots to identify inhibitory mechanisms. For solution plasminogen activation assays, histones were incubated with glu-plasminogen $(250 \mathrm{nM})$ and $\mathrm{tPA}(10 \mathrm{nM})$ in reaction buffer $(10 \mathrm{mM}$ Tris $\mathrm{pH} 7.7$, $100 \mathrm{mM} \mathrm{NaCl}, 0.01 \%$ tween-20), before addition of S-2251 ( $0.6 \mathrm{mM}$, final concentrations). Rates of plasminogen activation were measured by hydrolysis of S-2251 and calculated from plots of absorbance versus time squared using online apps. ${ }^{25}$ 


\section{Crosslinking Analysis}

For crosslinking analysis in purified systems, fibrinogen $(8.5 \mu \mathrm{M})$ was mixed with mixed histones and clotted for 2 hours at $37^{\circ} \mathrm{C}$ with thrombin $(5 \mathrm{nM})$ in $\mathrm{HBS}+5 \mathrm{mM} \mathrm{CaCl}_{2}$. Insoluble fibrin was isolated by centrifugation $(7,000 \mathrm{~g} /$ 10 minutes) and resuspended in clot solubilization buffer (0.2 mM Tris-HCl pH 8, $8 \mathrm{M}$ urea, $4 \%$ SDS, $40 \mathrm{mM}$ DTT) for 1 hour at $37^{\circ} \mathrm{C}$ and analyzed by sodium dodecyl sulfate polyacrylamide gel electrophoresis (SDS-PAGE) and Coomassie staining or western blotting. For time-course analysis of crosslinking, reactions were stopped at the indicated time points with clot solubilization buffer and analyzed as above.

Crosslinking assays with individual recombinant histones expressed with $\mathrm{N}$-terminal polyhistidine $\left(\mathrm{His}_{6}\right)$ tags were performed as above and allowed all histone subtypes to be identified with a single anti-his $s_{6}$ tag antibody. Citrullinated histones were prepared by incubating mixed histones with recombinant PAD4 as previously described, ${ }^{15}$ and used in crosslinking reactions as above.

For crosslinking analysis in plasma, sixfold diluted plasma (normal or FXIII-deficient) was clotted $\left(7.5 \mathrm{mM} \mathrm{CaCl}_{2}, 2 \mathrm{nM}\right.$ thrombin, final concentrations) in the presence of histones or T101. After 2 hours, clots were dissolved in solubilization buffer $\left(60^{\circ} \mathrm{C}, 1\right.$ hour $)$ and analyzed by western blot. For FXIIIreconstituted plasma, purified FXIII was added to FXIIIdeficient plasma prior to clotting.

Whole blood was clotted $\left(11.25 \mathrm{mM} \mathrm{CaCl}_{2}, 5 \mathrm{nM}\right.$ thrombin, final concentrations) in the presence of histones and/or T101 $(200 \mu \mathrm{M})$ for 2 hours at room temperature. Clots were collected by centrifugation ( $300 \mathrm{~g} / 15$ minutes) and homogenized in cell lysis buffer (10 mM HEPES pH 7.4, $150 \mathrm{mM} \mathrm{NaCl}$, $1 \mathrm{mM}$ EDTA, $1 \%$ Triton-X 100) using a Dounce homogenizer. Insoluble fibrin was isolated by centrifugation $(7,000 \mathrm{~g}$, 15 minutes, $4^{\circ} \mathrm{C}$ ) and the homogenization process repeated twice. The fibrin pellet was dissolved in clot solubilization buffer for 2 hours at $60^{\circ} \mathrm{C}$ and analyzed by SDS-PAGE and Coomassie staining or western blotting.

\section{Turbidimetric Analysis of Fibrin Clotting and Structure} Fibrinogen $(8.5 \mu \mathrm{M})$ was clotted with thrombin $(5 \mathrm{nM})$ in the presence of increasing histones with or without $\mathrm{CaCl}_{2}(5 \mathrm{mM})$ and/or T101 $(200 \mu \mathrm{M})$. Clotting was monitored for 2 hours in a Spectramax M5 plate reader (Molecular Devices, Berkshire, United Kingdom) at $405 \mathrm{~nm}$ at $37^{\circ} \mathrm{C}$. Times to $50 \%$ clotting were determined using online apps ${ }^{25}$ and slopes of the linear part of the clotting curve (which represent rates of protofibril lateral aggregation) determined according to Pieters et al. ${ }^{26}$ Relative mass-to-length ratios of the clots were determined from turbidity measurements as described by Carr and Hermans ${ }^{27}$ and updated by Yeromonahos and colleagues. ${ }^{28}$ Briefly, gels were scanned from 400 to $800 \mathrm{~nm}$ in a Spectramax M5 plate reader (example raw data are provided in - Supplementary Fig. S4A, available in the online version). Optical density values (D) were transformed to turbidity values using $\tau=1-e^{(-D .1 .1 n}$ (10)) . A plot of $\tau \lambda^{5}$ versus $\lambda^{2}$ produced accurate straight-line fits $\left(R^{2}>0.98\right)$, where the mass-to-length ratio was calculated from the slope (example data provided in -Supplementary Fig. S4B, available in the online version).

\section{Clot Lysis Turbidity Assays}

In microtiter plates, $75 \mu \mathrm{L}$ normal plasma (diluted twofold with $1.5 \mathrm{nM}$ tPA and $25 \mathrm{mM} \mathrm{CaCl}_{2}$ ) was mixed with $25 \mu \mathrm{L}$ histones and thrombin ( $5 \mathrm{nM}$ final) in HBS. For FXIII-reconstituted plasma, FXIII was added to FXIII-deficient plasma to $1 \mathrm{IU} / \mathrm{mL}$ and clotted as above. For clot lysis assays with purified fibrinogen, fibrinogen $(8.5 \mu \mathrm{M})$ was mixed with glu-plasminogen $(90 \mathrm{nM})$, tPA $(0.6 \mathrm{nM})$, LMWH $(1 \mathrm{IU} / \mathrm{mL})$, and histones, and clotted with thrombin $(5 \mathrm{nM})$ in $\mathrm{HBS}+5 \mathrm{mM} \mathrm{CaCl}$. Clotting and lysis were monitored in a Spectramax M5 plate reader (Molecular Devices, Berkshire, United Kingdom) at $340 \mathrm{~nm}$ (plasma) or $405 \mathrm{~nm}$ (fibrinogen) and analyzed using online apps to determine times to $50 \%$ lysis. ${ }^{25}$

For direct lysis of clots by plasmin, plasmin $(250 \mathrm{nM})$ was added to the surface of clots and lysis monitored by the decrease in absorbance at $405 \mathrm{~nm}$. Absorbance values were normalized to account for different starting turbidities of clots containing histones, which was independent of fibrin concentration (normalized absorbance at $405 \mathrm{~nm}, \mathrm{nAbs}_{405}=\left(A-A_{0}\right) /$ $A_{\max }$, where $A_{\max }$ is the maximal and $A_{0}$ the final absorbance after complete lysis). In parallel plates, clots were dissolved in clot solubilization buffer at different time points following application of plasmin. Samples were analyzed by SDS-PAGE and Coomassie staining and band intensities quantitated using ImageJ software (ImageJ, National Institute of Health, United States).

\section{Rotational Thromboelastometry}

Viscoelastic measurement of blood was performed with a rotational thromboelastometry (ROTEM) delta (Werfen, Warrington, United Kingdom), operated according to the manufacturer's instructions. Blood was collected from local donors, with approval of the local ethical committee, and mixed 9 parts blood to 1 part $3.2 \%$ sodium citrate. Blood was mixed with histones, T101 $(200 \mu \mathrm{M})$, and tPA $(2.5 \mathrm{nM})$, and incubated at $37^{\circ} \mathrm{C}$ for 5 minutes prior to activation with EXTEM (extrinsically activated thromboelastometric test) reagents supplied by the manufacturer.

\section{Statistical Methods}

Means and standard deviations were calculated using GraphPad Prism. The effects of histones on clotting time, lateral aggregation, and fibrin mass/length were analyzed by one-way ANOVA (analysis of variance) with Dunnett's or Tukey's multiple comparison testing in GraphPad Prism.

\section{Results}

\section{Histones Inhibit Plasmin to Delay Fibrinolysis}

The effect of mixed histones, consisting of the four core histone subtypes (H2A, $\mathrm{H} 2 \mathrm{~B}, \mathrm{H} 3$, and $\mathrm{H} 4$ ) plus the $\mathrm{H} 1$ linker histone, on the activity of plasmin and tPA is shown in - Fig. 1A. Plasmin hydrolysis of the chromogenic substrate S-2251 was inhibited by histones, with an $\mathrm{IC}_{50}$ of $11.5 \mu \mathrm{g} / \mathrm{mL}$. By contrast, histones had little effect on the activity of tPA on its substrate S-2288 (-Fig. 1A). Inhibition of plasmin was competitive; mixed histones increased the $K_{\mathrm{m}}$ of plasmin for S-2251 up to seven-

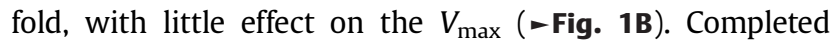


A

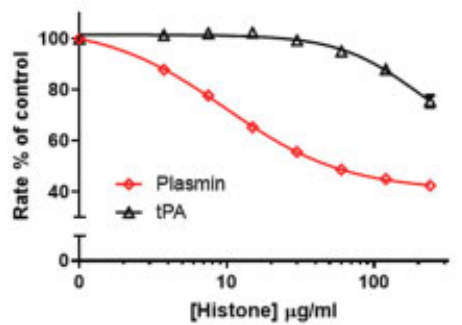

D

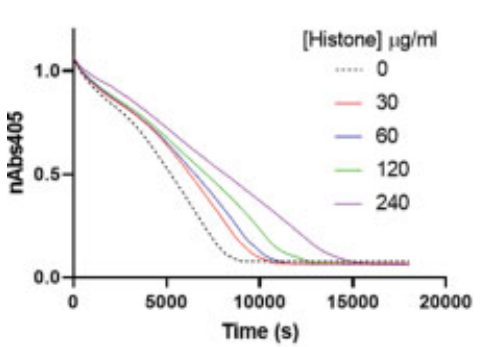

B

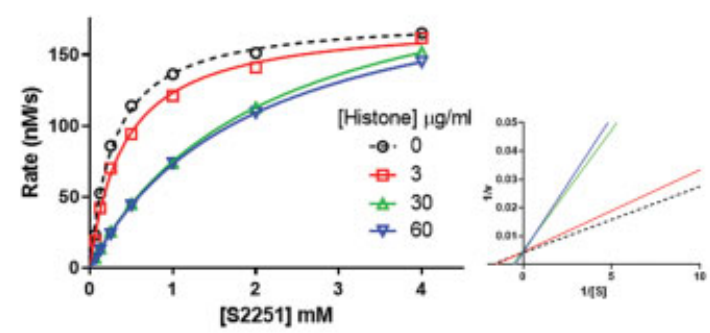

E

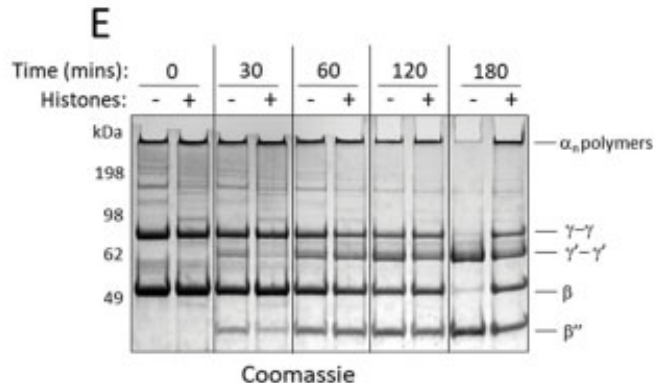

H

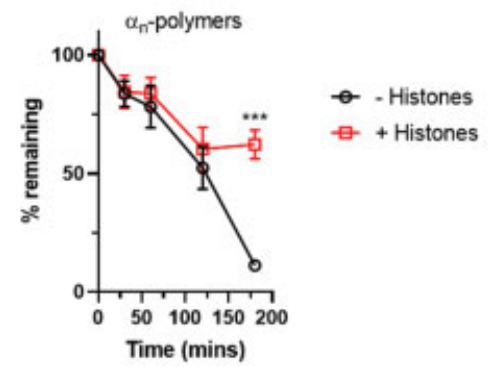

C

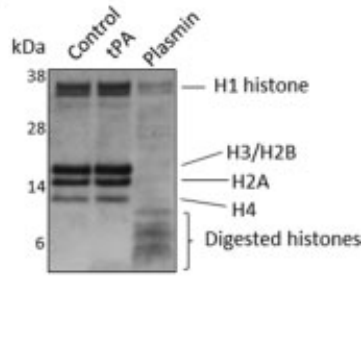

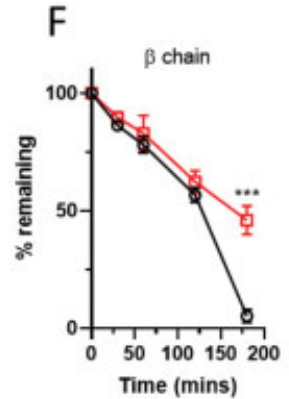

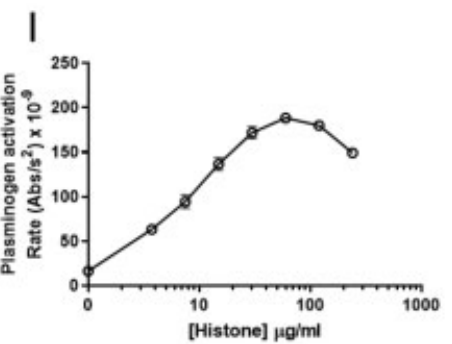

G

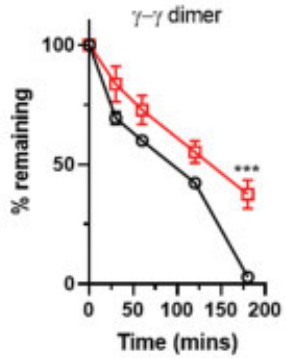

J

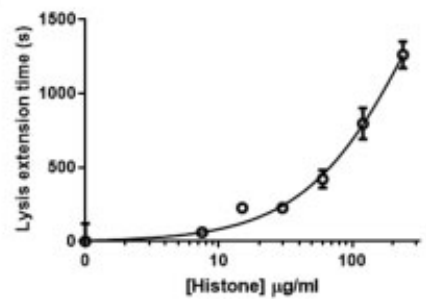

Fig. 1 Histones inhibit plasmin to delay fibrinolysis in a purified system. (A) tPA or plasmin (5 nM) was incubated with S-2288 or S-2251, respectively, and the indicated concentration of mixed histones. Initial rates were calculated from plots of absorbance ( $405 \mathrm{~nm})$ versus time and expressed relative to the condition without added histone. Points represent means \pm SEM from duplicate wells. (B) Plasmin activity was measured with increasing amounts of S-2251 in the presence of the indicated histones. The inset shows a Lineweaver-Burk plot to illustrate competitive inhibition of plasmin by histones. (C) Completed reactions from (A) for $240 \mu \mathrm{g} / \mathrm{mL}$ histones were analyzed by SDS-PAGE and Coomassie staining. $\mathrm{H} 1$ histone, core complex ( $\mathrm{H} 2 \mathrm{~A}, \mathrm{H} 2 \mathrm{~B}, \mathrm{H} 3$, and $\mathrm{H} 4)$, and digested histones are annotated. The control sample represents histones without tPA or plasmin incubation. (D) Plasmin was added to the surface of fibrin clots prepared by mixing fibrinogen, thrombin, and $\mathrm{Ca}^{2+}$ with the indicated concentration of histone. Fibrinolysis was monitored by absorbance $(405 \mathrm{~nm})$ and turbidity values normalized as described in the Materials and Methods section. Lysis curves represent means of three experiments. (E) Fibrin clots with or without histones $(240 \mu \mathrm{g} / \mathrm{mL})$ were incubated with plasmin and solubilized at the indicated time points for reducing SDS-PAGE and Coomassie staining. The positions of fibrin(ogen) $\alpha, \beta$, and $\gamma$ chains and crosslinked variants ( $\gamma-\gamma$ dimer and $\alpha_{n}$-polymers), together with digestion products of $\gamma-\gamma$ $\operatorname{dimer}\left(\gamma^{\prime}-\gamma^{\prime}\right)$ and $\beta$ chain $\left(\beta^{\prime \prime}\right)$, are annotated. Bands corresponding to the $\beta$ chain $(F), \gamma-\gamma \operatorname{dimer}(\mathbf{G})$, and $\alpha_{n}$ polymers $(H)$ were quantitated and expressed relative to their starting intensities. Data represent mean $\pm \operatorname{SEM}(n=3) .{ }^{* * *} p<0.001$ (I). Rates of plasminogen activation by tPA in the presence of increasing histones were measured by hydrolysis of S-2251 and calculated from plots of absorbance versus time squared. Points shown are means \pm SEM from duplicate wells. (J) Internal clot lysis assays catalyzed by tPA were performed by clotting purified fibrinogen with thrombin in the presence of increasing histones, $\mathrm{Ca}^{2+}$, plasminogen, and tPA. Inhibition of fibrinolysis by histones is expressed as extension to $50 \%$ lysis times. Errors bars are \pm SEM from duplicate wells. SEM, standard error of mean; tPA, tissue type-plasminogen activator. 
reactions from - Fig. 1A were analyzed by SDS-PAGE and Coomassie staining, revealing that $\mathrm{H} 1$ and core histones were digested by plasmin, but not by tPA ( - Fig. 1C). To determine the effect of histones on plasmin-mediated fibrinolysis, plasmin was applied to the surface of preformed clots containing histones (-Fig. 1D). In this purified system, histones dosedependently delayed fibrin digestion. Protection of fibrin by histones could be visualized by stopping fibrinolysis at regular intervals during incubation with plasmin and solubilizing the clot for SDS-PAGE analysis ( - Fig. 1E). Compared with plasmin lysis in the absence of histones, the presence of histones slowed digestion of bands corresponding to the $\beta$ chain ( - Fig. 1F), $\gamma-\gamma$ dimer (-Fig. 1G), and $\alpha_{n}$ polymers (-Fig. 1H).

As histones bind plasminogen, ${ }^{29}$ we examined whether they influenced plasminogen activation by tPA. Low concentrations of histones stimulated plasmin generation by tPA but became inhibitory at higher concentrations, resulting in a bell-shaped dose-response curve (-Fig. 1I). Despite these mixed effects on plasminogen activation in solution, histones inhibited fibrinolysis catalyzed by tPA in a purified clot lysis system and extended 50\% lysis times at all concentrations examined (-Fig. 1 J).

\section{Histones Are Crosslinked to Fibrin by FXIIla}

Histones are rich in lysine residues which could potentially serve as amine donors in transglutaminase reactions. ${ }^{19}$ This prompted us to investigate whether histones could be covalently incorporated into fibrin by FXIIIa.

Commercial fibrinogen, which has contaminating FXIII activity (-Supplementary Fig. S1, available in the online version), was clotted with thrombin in the presence of increasing amounts of mixed histone $(30-120 \mu \mathrm{g} / \mathrm{mL})$ under crosslinking $\left(+\mathrm{Ca}^{2+}\right)$ or noncrosslinking conditions $\left(-\mathrm{Ca}^{2+}\right.$ or + T101, a FXIIla inhibitor). Fibrin was isolated from the clot milieu by centrifugation and solubilized for analysis. Noncovalently and covalently bound histones were detected by western blot with antibodies specific for histone $\mathrm{H} 3$, which we used as a representative member of the core histone complex previously identified in patient thrombi. ${ }^{8}$ Histone H3 co-purified as a noncovalently bound monomer $(\sim 14 \mathrm{kDa})$ with noncrosslinked fibrin $\left(-\mathrm{Ca}^{2+}\right.$ or $+\mathrm{T} 101$ in -Fig. 2A). Under crosslinking conditions $\left(+\mathrm{Ca}^{2+}\right.$, which is required for dissociation of the inhibitory FXIII-B subunits from the catalytic FXIII-A subunits), the intensity of the histone $\mathrm{H} 3$ band was reduced and replaced by high molecular weight histone H3 immunoreactivity, beginning just above the position of the $\gamma-\gamma$ dimer $(\sim 62 \mathrm{kDa})$. This indicated histone $\mathrm{H} 3$ had been crosslinked to fibrin, resulting in a heterogeneous mixture of histone-fibrin crosslinked polymers. High molecular weight histone-fibrin crosslinking was not apparent when $\mathrm{Ca}^{2+}$ was omitted or T101 was included, indicating the involvement of FXIIIa.

A time-course analysis of histone-fibrin and fibrin-fibrin crosslinking is shown in - Fig. 2B. Distinct bands of histone H3 ( $\sim 70 \mathrm{kDa}$ and $\sim 100 \mathrm{kDa}$ ) could be detected approximately 5 to 10 minutes after initiation of clotting, which roughly correlates to the molecular masses of the $\alpha$-chain of fibrin and crosslinked $\gamma-\gamma$ dimer. Interestingly, higher molecular weight histone H3 immunoreactivity ( $>98 \mathrm{kDa}$ ) was detected only after $\gamma-\gamma$ dimer formation was complete (120 minutes; -Fig. 2B). It is possible that histone H3 immunoreactivity could originate, at least in part, from histones crosslinked to one another, which subsequently co-purified with fibrin. A band approximately $30 \mathrm{kDa}$ in size was apparent in - Fig. 2B, which is a potential crosslinked histone dimer. However, the distinctive banding pattern of histone $\mathrm{H} 3$ early in the time course suggests that histones are conjugated to crosslinked fibrin.

We extended our crosslinking analysis by testing individual recombinant histones. Results in - Supplementary Fig. S2 (available in the online version) show that all histone subtypes (H1, H2A, H2B, H3, and H4) could be crosslinked to fibrin in a FXIIla-dependent manner. Bands corresponding to histone dimers were apparent for histones $\mathrm{H} 2 \mathrm{~A}$ and $\mathrm{H} 3$, but these were also observed in the presence of T101, and so the exact nature of these histone oligomers and whether they are crosslinked forms require further study.

Histones in NETs may be citrullinated by PAD4 during their release from the cell, potentially altering histone interactions. To determine whether citrullinated histones could still bind fibrin, we repeated our crosslinking analysis with citrullinated histones, generated in vitro by incubating histones with recombinant PAD4. Similar to unmodified histones, citrullinated histone $\mathrm{H} 3$ co-purified with fibrin in noncovalently bound and high molecular weight crosslinked forms, with crosslinking being dependent on FXIIIa (-Supplementary Fig. S3, available in the online version).

To show that histones could be crosslinked to fibrin in more complex and physiologically relevant environments, plasma was clotted in the presence of histones and T101. With clotting (i.e., recalcification) crosslinked fibrin $(\gamma-\gamma$ dimers and high molecular weight polymers) was detected, but not in the presence of T101, demonstrating effective FXIIla inhibition (-Fig. 2C). High molecular weight histone $\mathrm{H} 3$ was again detected in clots, but not when FXIIIa was inhibited. Supporting FXIIIa involvement, fibrin-fibrin and fibrin-histone crosslinking was not detected in clots from FXIII-deficient plasma, but was restored by the addition of purified FXIII to normal plasma levels (-Fig. 2D).

Analysis of fibrin isolated from whole blood clots, clotted in the presence of mixed histones, is presented in - Fig. $2 \mathbf{E}$. Inclusion of T101 resulted in loss of $\gamma-\gamma$ dimers and $\alpha_{n}$ polymers and the reappearance of $\alpha$ and $\gamma$ chains, demonstrating effective FXIIIa inhibition in blood ( - Fig. 2E). The histone H3 western blot shows co-purification of histone H3 ( 14 kDa) and high molecular weight histone $\mathrm{H} 3$ immunoreactivity, which was prevented by FXIIIa inhibition. Inhibition of crosslinking with T101 coincided with increased intensity of noncovalently bound histone H3 monomer, consistent with histones binding fibrin without being crosslinked.

\section{Histones Accelerate Fibrin Polymerization and Enhance Clot Structure Independent of Crosslinking to Fibrin}

Binding of histones to fibrin(ogen) alters fibrin polymerization and fibrin network structure. ${ }^{17,24}$ To determine whether these effects are dependent on histone-fibrin crosslinking, we clotted 

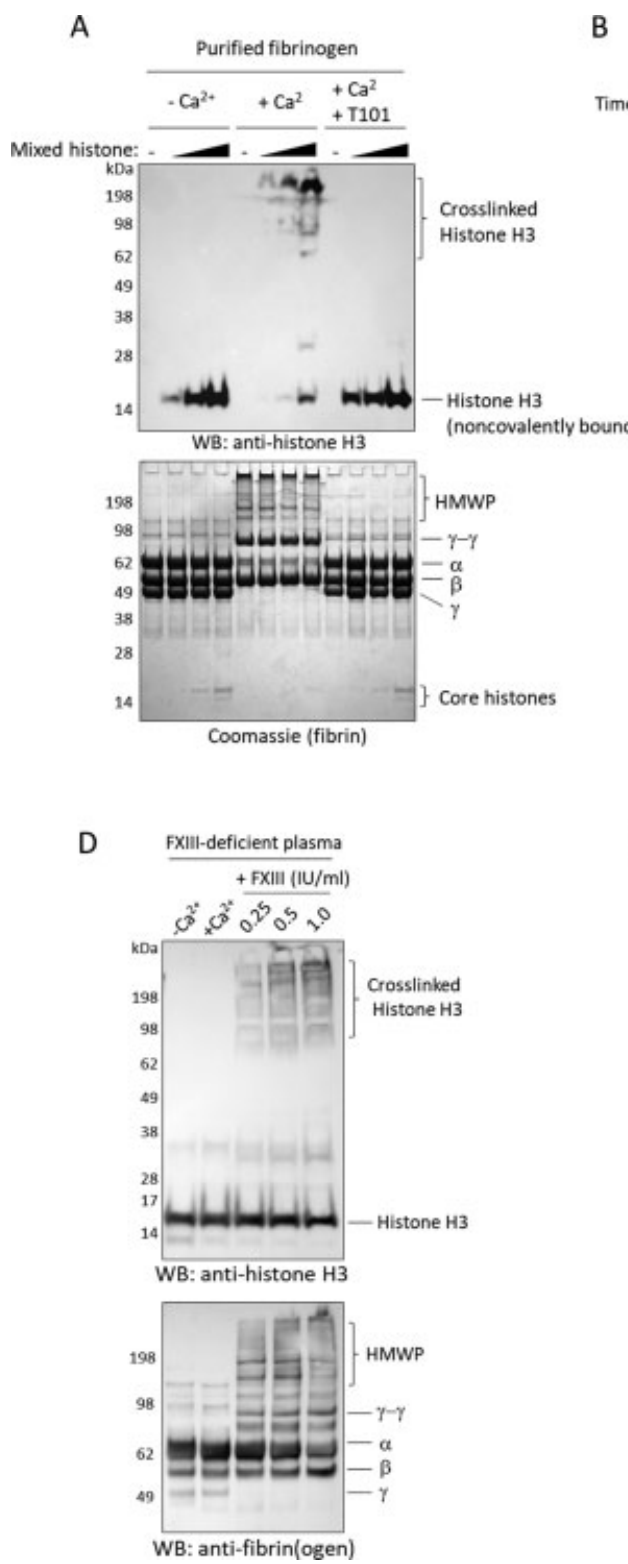

B

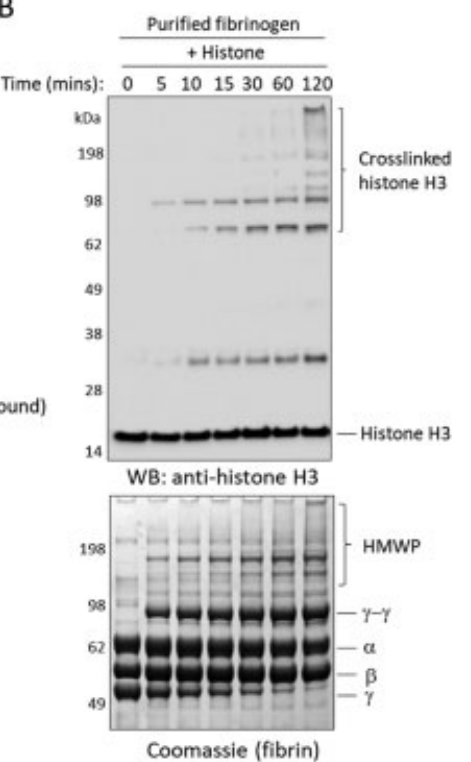

E

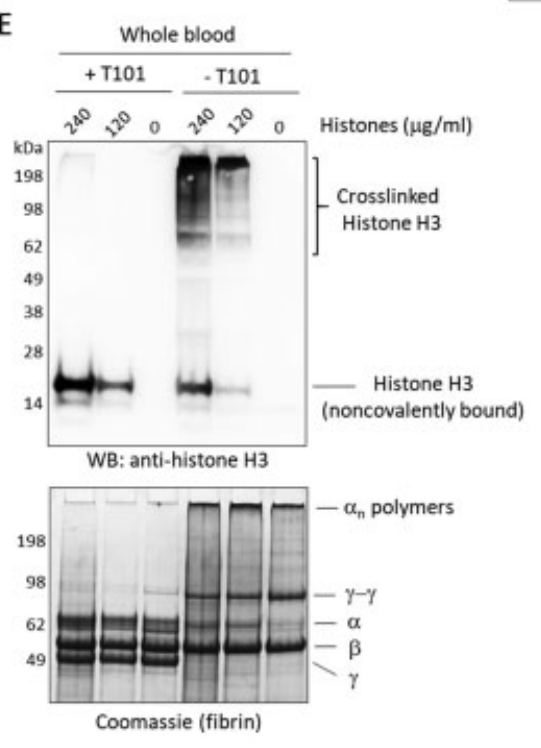

C
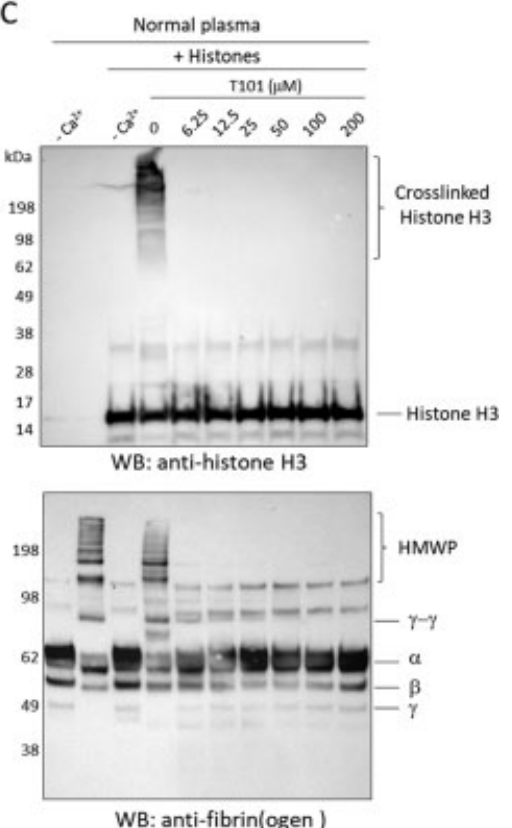

WB: anti-fibrin(ogen )

Fig. 2 Histones are crosslinked to fibrin in purified, plasma, and whole blood systems. (A) Purified fibrinogen was mixed with thrombin in the presence or absence of $\mathrm{Ca}^{2+}$, FXIlla inhibitor T101 $(200 \mu \mathrm{M})$, and increasing amounts of histones $(30,60$, or $120 \mu \mathrm{g} / \mathrm{mL})$. Insoluble fibrin was isolated from the clot by centrifugation and solubilized for analysis by SDS-PAGE and Coomassie staining (bottom panel) or western blotting with anti-histone $\mathrm{H} 3$ antibodies (upper panel). The positions of crosslinked and noncrosslinked fibrin(ogen) are annotated on the Coomassie gel. The expected positions of noncovalently bound, monomeric histone $\mathrm{H3}$ ( $\sim 14 \mathrm{kDa}$ ) and high molecular weight crosslinked forms are annotated on the western blot. (B) Fibrin clots were prepared as in (A) in the presence of mixed histones $(120 \mu \mathrm{g} / \mathrm{mL})$ and clotting stopped at the indicated times. (C) Histones $(240 \mu \mathrm{g} / \mathrm{mL})$ and T101 were added to clotting plasma. After 2 hours, clots were dissolved in solubilization buffer and analyzed by western blot with antibodies specific for fibrin(ogen) and histone $\mathrm{H} 3$. The positions of crosslinked fibrin and histone are annotated. (D) FXIII-deficient plasma was incubated with histones $(240 \mu \mathrm{g} / \mathrm{mL}$ ) and clotted as in (A), with or without addition of purified FXIII (0.25-1.0 IU/mL). Crosslinked species were detected as in (A). (E) Whole blood was clotted in the presence of the indicated concentration of histone and/or T101 (200 $\mu \mathrm{M})$ for 2 hours at room temperature. Clots were collected by centrifugation followed by homogenization/washing to obtain the isolated fibrin component, which was dissolved and analyzed by SDS-PAGE and Coomassie staining or western blotting. Fibrin-fibrin and histone-fibrin crosslinked products are annotated.

purified fibrinogen with thrombin in the presence of histones, with or without $\mathrm{Ca}^{2+}$ and/or FXIIla inhibitor (T101). Raw clotting curves are presented in - Fig. $\mathbf{3 ( A - C )}$ illustrating that histones shortened clotting times (time to 50\% maximum absorbance, - Fig. 3D) and increased rates of lateral aggregation of protofibrils, defined by the slope of the turbidimetric curve ( - Fig. 3E). These effects were unaffected by the omission of $\mathrm{Ca}^{2+}$ ( - Fig. 3B and red bars in - Fig. 3D, E) or the presence of T101 (-Fig. 3C and green bars in -Fig. 3D, E).
Histones increased the maximum optical density of fibrin clots ( - Fig. 3A-C), which reflects the mass-to-length ratio of fibrin fibers. ${ }^{27}$ Mass-to-length measurements were calculated from turbidity (see the Materials and Methods section and $\boldsymbol{\sim}$ Supplementary Fig. $\mathbf{S 4}$ [available in the online version]) and the results are presented in - Fig. 3F. Histones increased fibrin fiber mass-to-length ratios in a dose-dependent manner, reaching a maximum of approximately 2.2 -fold over the concentration range studied, indicative of thicker fibrin fibers, 
A

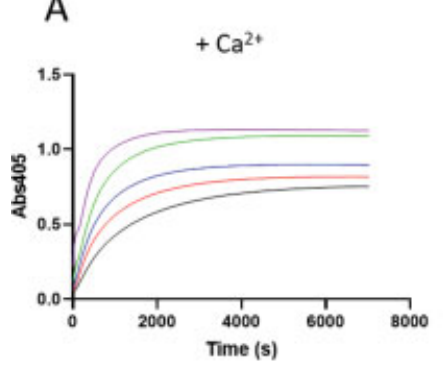

D

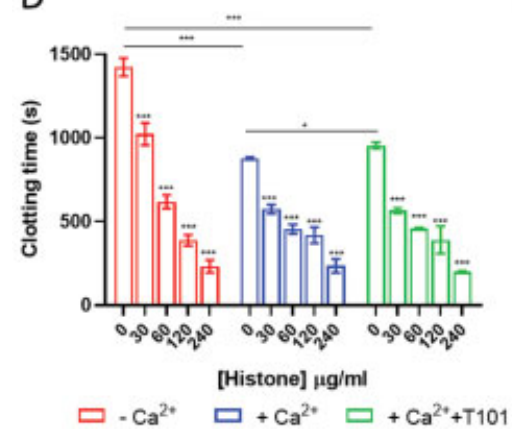

B

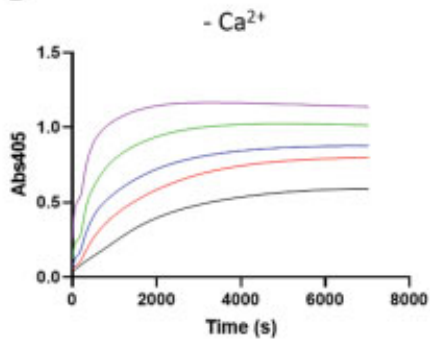

$\mathrm{E}$

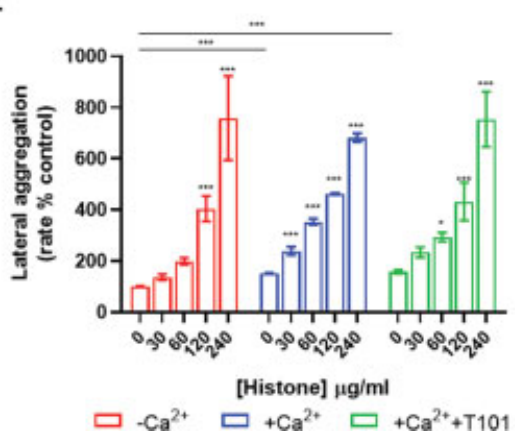

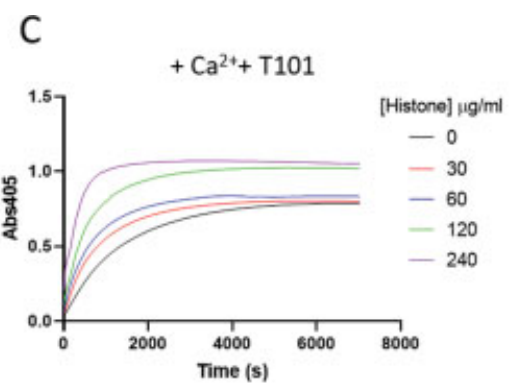

$\mathrm{F}$

Fig. 3 Histones accelerate fibrin polymerization and enhance clot structure independent of crosslinking to fibrin. (A-C) Purified fibrinogen was clotted by thrombin in the presence of the indicated amounts of histone in buffer containing $\mathrm{Ca}^{2+}(\mathrm{A})$, without $\mathrm{Ca} \mathrm{a}^{2+}(\mathrm{B})$, or containing T101 $(200 \mu \mathrm{M})$ plus $\mathrm{Ca}^{2+}(\mathrm{C})$. Fibrin formation was monitored by absorbance $(405 \mathrm{~nm})$. Curves are average of triplicate measurements. (D) Time to $50 \%$ clotting (half-maximum absorbance), (E) rates of lateral aggregation of protofibrils, and (F) fibrin fiber mass-to-length ratios calculated from turbidity measurements from A-C (see the Materials and Methods section and - Supplementary Fig. S4 [available in the online version] for details). Rates in (E) are relative to clots without $\mathrm{Ca}^{2+}$ and histones. Error bars in D-F represent $95 \%$ confidence intervals of the mean from three measurements. Statistical analysis was performed using one-way ANOVA and is relative to conditions without histones. ${ }^{*} p<0.05,{ }^{* * *} p<0.001$. ANOVA, analysis of variance.

as previously described. ${ }^{17}$ Similar results were obtained in the absence of $\mathrm{Ca}^{2+}$ or the presence of T101, suggesting histonefibrin crosslinking was not required for these effects. Consistent with previous studies, $\mathrm{Ca}^{2+}$ and $\mathrm{T} 101$ had subtle but significant effects on fibrin-fiber thickness in the absence of histones (-Fig. 3F). ${ }^{30,31}$

\section{The Antifibrinolytic Potency of Histones Is Enhanced by Crosslinking to Fibrin}

Clot lysis profiles examining the effect of histones and FXIII on tPA-mediated fibrinolysis in normal, FXIII-deficient, and FXIIIreconstituted plasmas are shown in - Fig. $\mathbf{4}(\mathbf{A}-\mathbf{C})$, and the calculated extension to $50 \%$ clot lysis times in - Fig. 4D. Consistent with our results in purified systems (- Fig. 1J), histones delayed fibrinolysis and extended clot lysis times in normal plasma ( - Fig. 4A, D). However, histones were less effective at delaying fibrinolysis in FXIII-deficient plasma, causing only a modest extension to lysis times (-Fig. 4B, D). Reconstitution of FXIII activity by the addition of purified FXIII to FXIIIdeficient plasma restored the antifibrinolytic potency of histones to that measured in normal plasma ( - Fig. 4C, D).

The relationship between FXIII and histones was explored in whole blood using ROTEM, which measures viscoelastic clot strength over time. To simulate internal fibrinolysis, blood was spiked with tPA prior to clotting, which resulted in complete lysis after approximately 35 minutes (presented in the TEMograms in - Fig. 4E). The importance of FXIIIa for clot strength and stability was apparent with inclusion of T101, which significantly reduced clot strength and lysis times (-Fig. 4E-G). Addition of histones stabilized clots, and 60 and $120 \mu \mathrm{g} / \mathrm{mL}$ histones significantly delayed lysis (-Fig. 4F). Histones were less effective at stabilizing clots in the presence of T101, but still delayed clot lysis, particularly at $120 \mu \mathrm{g} / \mathrm{mL}$ ( - Fig. 4F). Histones also caused small but significant increases in clot strength with or without FXIIIa inhibition (-Fig. 4G).

\section{Low-Molecular-Weight Heparin Prevents Histone-fibrin Crosslinking and Improves Lysis of Clots Containing Histones}

Cationic histones have high affinity for heparin, which carries a strong negative charge. ${ }^{32}$ This led us to investigate whether LMWH could interfere with histone-fibrin crosslinking and their inhibitory effects on fibrinolysis.

Therapeutic doses of LMWH prevented histone-fibrin crosslinking in clots formed from purified fibrinogen, without affecting fibrin-fibrin crosslinking ( - Fig. 5A). LMWH at $0.5 \mathrm{IU} / \mathrm{mL}$ prevented crosslinking of $60 \mu \mathrm{g} / \mathrm{mL}$ histone and a higher dose of $1 \mathrm{IU} / \mathrm{mL}$ inhibited crosslinking of $120 \mu \mathrm{g} / \mathrm{mL}$ histone. Prevention of histone-fibrin crosslinking by LMWH coincided with increased noncovalently bound histone ( $\sim 14 \mathrm{kDa})$ and LMWH-accelerated lysis of clots containing histones in a purified clot lysis system ( - Fig. 5B-D). Histones increased the maximum turbidity of the clot lysis curves (-Fig. 5B, E), as previously noted (-Fig. 3), but not in the presence of LMWH (- Fig. $\mathbf{5 C}$, E). On the contrary, histones 
A

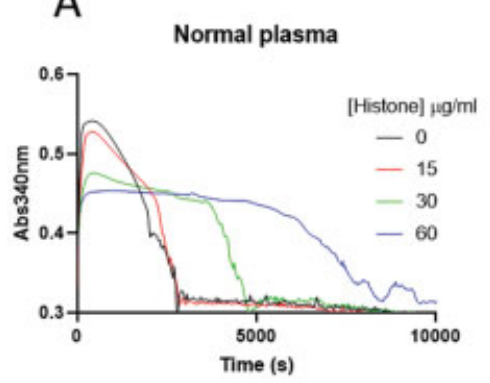

D

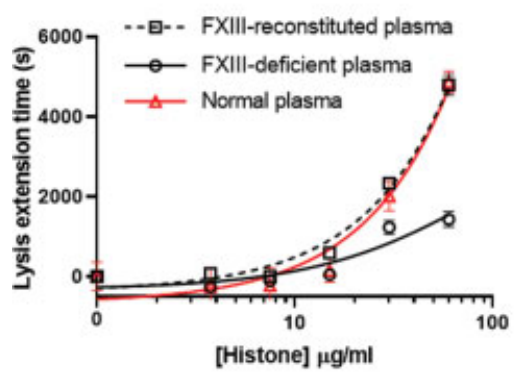

F

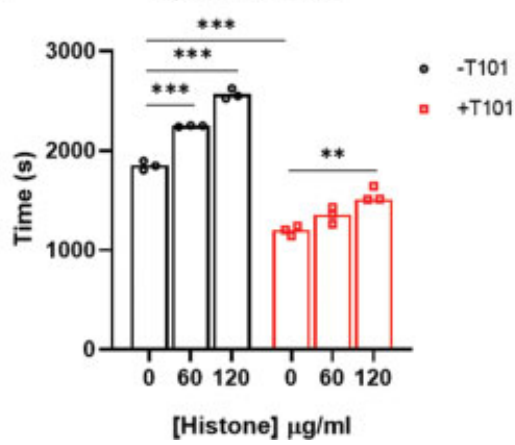

E
B

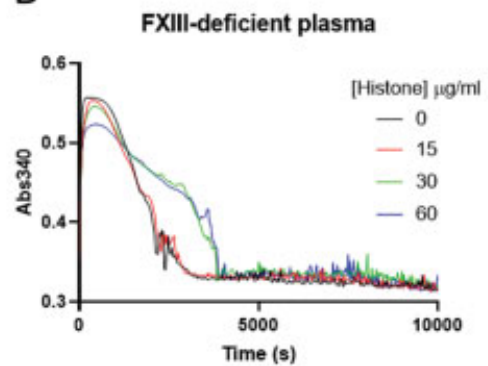

C

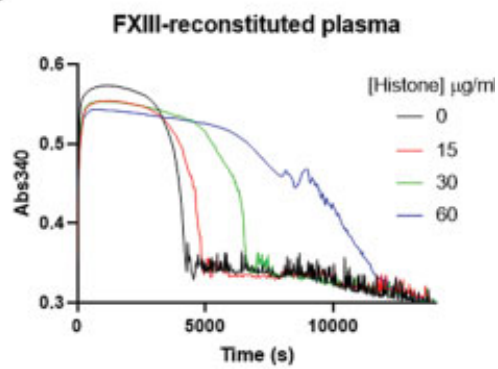

Histones:

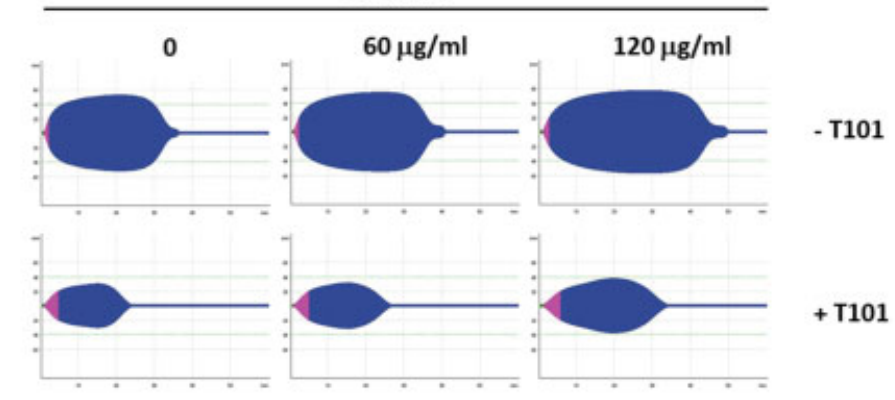

G

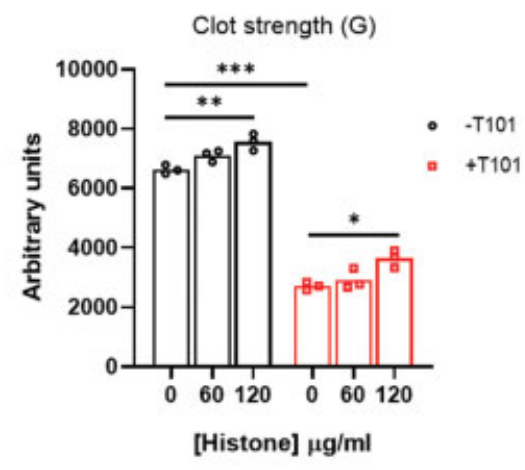

Fig. 4 The antifibrinolytic potency of histones is enhanced by crosslinking to fibrin. (A-C) Normal plasma, FXIII-deficient plasma, or FXIIIdeficient plasma supplemented with $1 \mathrm{IU} / \mathrm{mL}$ purified FXIIII were clotted in the presence of the indicated histone and $0.6 \mathrm{nM}$ tPA. Clotting and lysis were monitored at $340 \mathrm{~nm}$. Curves are averages of three experiments. (D) Extension to $50 \%$ lysis times by histones calculated from clot lysis curves in A-C. Error bars are \pm SEM from three experiments. (E) Citrated blood was incubated with histones, tPA (2.5 nM), and T101 (200 $\mu$ M), and clotting initiated by extrinsic pathway activation (EXTEM). Viscoelastic clot strength was monitored by rotational thromboelastometry (ROTEM) and is represented as clot firmness ( $y$-axis, arbitrary units) over time ( $x$-axis, minutes). ( $F$ ) Lysis onset time (defined as the time taken for maximum clot firmness to decrease by $15 \%$ ) and $(G)$ clot strength (defined by $G$, shear strength) derived from the TEMograms in (E). Each bar indicates the mean from the three measurements represented by each dot. Statistical analysis was performed using one-way ANOVA. Asterisks represents difference from "zero histone" control $\pm \mathrm{T} 101 .{ }^{*} p<0.05,{ }^{* *} p<0.01,{ }^{* * *} p<0.001$. ANOVA, analysis of variance; SEM, standard error of mean; tPA, tissue type-plasminogen activator.

reversed the increased clot turbidity caused by LMWH up to a concentration of $120 \mu \mathrm{g} / \mathrm{mL}$, after which turbidity began to increase (-Fig. 5E).

\section{Discussion}

A growing body of evidence implicates histones and NETs in the pathophysiology of thrombosis by promoting coagulation and inhibiting fibrinolysis. ${ }^{6-8}$ In this study, we show that histones stabilize fibrin through several mechanisms, summarized in - Fig. 6. By acting as competitive plasmin substrates histones protect fibrin from degradation, which is enhanced by covalent crosslinking of histones to fibrin, catalyzed by transglutaminase FXIIIa. Accordingly, fibrinolysis is enhanced by blocking histone-fibrin crosslinking with FXIIla inhibitors (-Fig. 4) or LMWH ( - Fig. 5). Noncovalently bound histones accelerate lateral aggregation of protofibrils, resulting in thicker fibrin fibers with higher mass-to-length ratios (-Fig. 3). In the absence of FXIIla activity, histones have a more modest effect on fibrinolysis but still enhance clot strength ( - Fig. 4). Together, these results show that by interacting noncovalently and covalently with fibrin, histones inhibit fibrinolysis and enhance clot stability.

Histone-fibrinogen interactions have been studied via several approaches including turbidimetric, proteomic, and biophysical methods. ${ }^{13-15,17}$ However, the exact histone- 
A
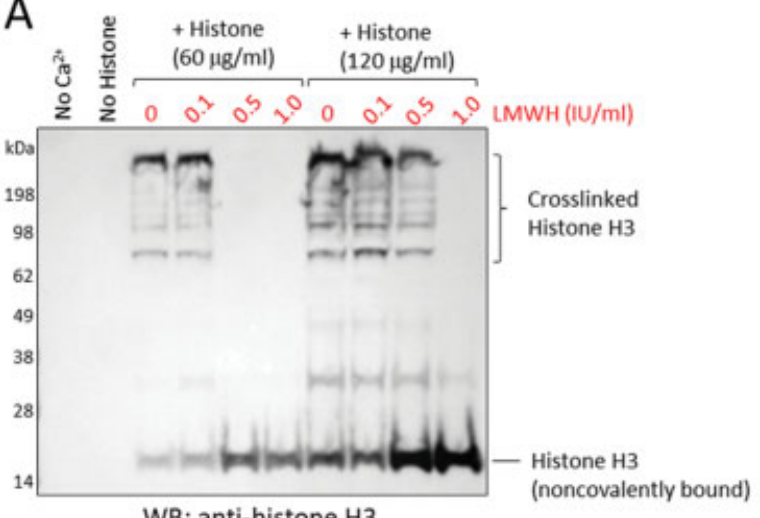

WB: anti-histone $\mathrm{H} 3$

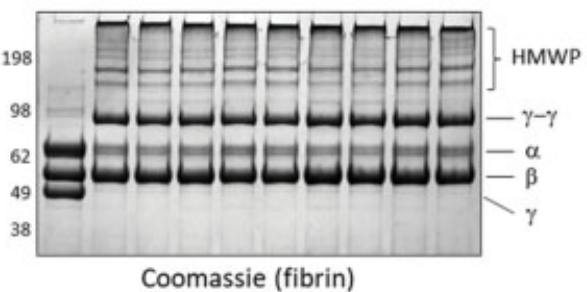

D

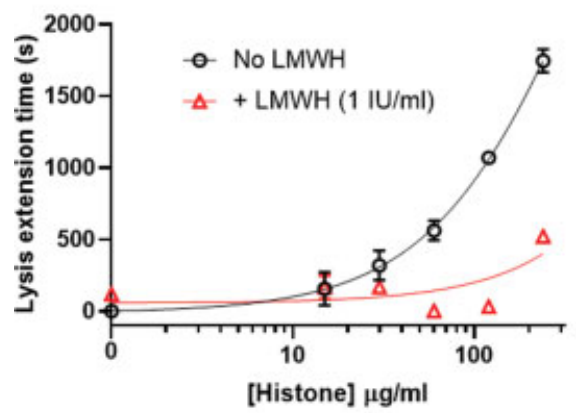

B

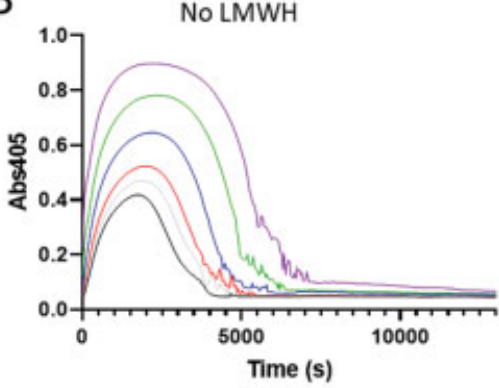

C

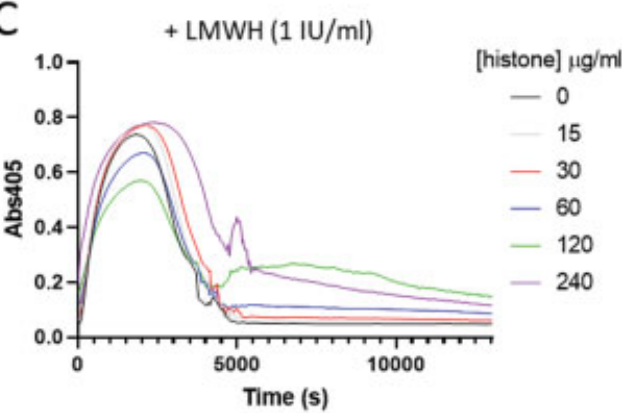

E

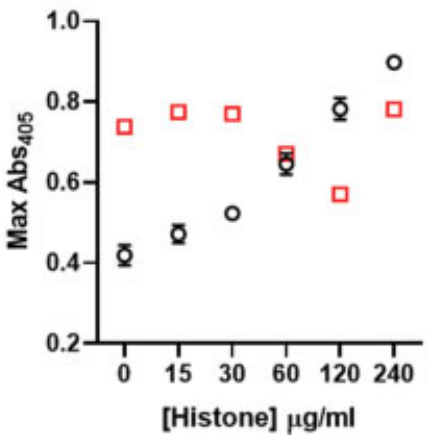

- No LMWH

ㅁ + LMWH (1 IU/ml)

Fig. 5 Low-molecular-weight heparin (LMWH) prevents histone-fibrin crosslinking and neutralizes the antifibrinolytic potency of histones. (A) Fibrinogen was clotted by thrombin in the presence of the indicated concentration of histone and LMWH for 2 hours, followed by isolation of insoluble fibrin from the clot by centrifugation. Fibrin was solubilized and analyzed by SDS-PAGE and Coomassie staining or western blotting. (B, C) Raw clot lysis profiles of fibrinogen clotted with thrombin in the presence of plasminogen, $\mathrm{PAA}, \mathrm{Ca}^{2+}$, and the indicated histone without (B) or with $1 \mathrm{IU} / \mathrm{mL}$ LMWH (C). (D) Extension to $50 \%$ clot lysis times by histones calculated from clot lysis curves in B and C. (E) Maximum turbidity of clot lysis curves in B and C. Error bars in D and $E$ represent \pm SEM from duplicate measurements. SEM, standard error of mean.

binding site(s) on fibrin(ogen) have yet to be determined. It is possible that electrostatic forces mediate the binding of positively charged histones to fibrinogen, which is predicted to contain uneven regions of positive and negative charges distributed on the D- and E-domains. ${ }^{33}$ When mixed, solutions of histones and fibrinogen form gels that can be studied turbidimetrically and we recently showed that fibrinogen gelation and accelerated clotting are dependent on histone positive charge. ${ }^{15}$ In the current work, citrullinated histones, which have reduced positive charge due to conversion of arginine to citrulline, retained their ability to bind fibrin (-Supplementary Fig. S3, available in the online version). This suggests histone-fibrin interactions might be more than simply electrostatic, and hydrophobic interactions may contribute. ${ }^{13}$ We also found that histones increased the rate of fibrin protofibril aggregation and enhanced clot structure without being crosslinked to fibrin
(-Fig.3). Consistent with this, previous electron microscopy and small-angle X-ray scattering experiments coupled with rheological studies showed that histones enhanced fibrin structure and mechanical stability in the absence of FXIIIa activity. ${ }^{17}$ Taken together, our results suggest that noncovalent interactions between histones and fibrin(ogen) alter the structural and mechanical properties of fibrin.

Plasmin is a broad specificity serine protease with a preference for peptide bonds adjacent to arginine and lysine residues, making histones good candidate targets. We found that all histone subtypes could be digested by plasmin and competed with fibrin and synthetic chromogenic plasmin substrates (-Fig. 1). A wide range of histone concentrations inhibited fibrinolysis by plasmin ( - Fig. 1D) and tPA ( - Fig. 1)). However, in solution a narrow range of histones appeared to stimulate plasminogen activation by tPA, which plateaued and became inhibitory (-Fig. 1I). The interaction between histones and 


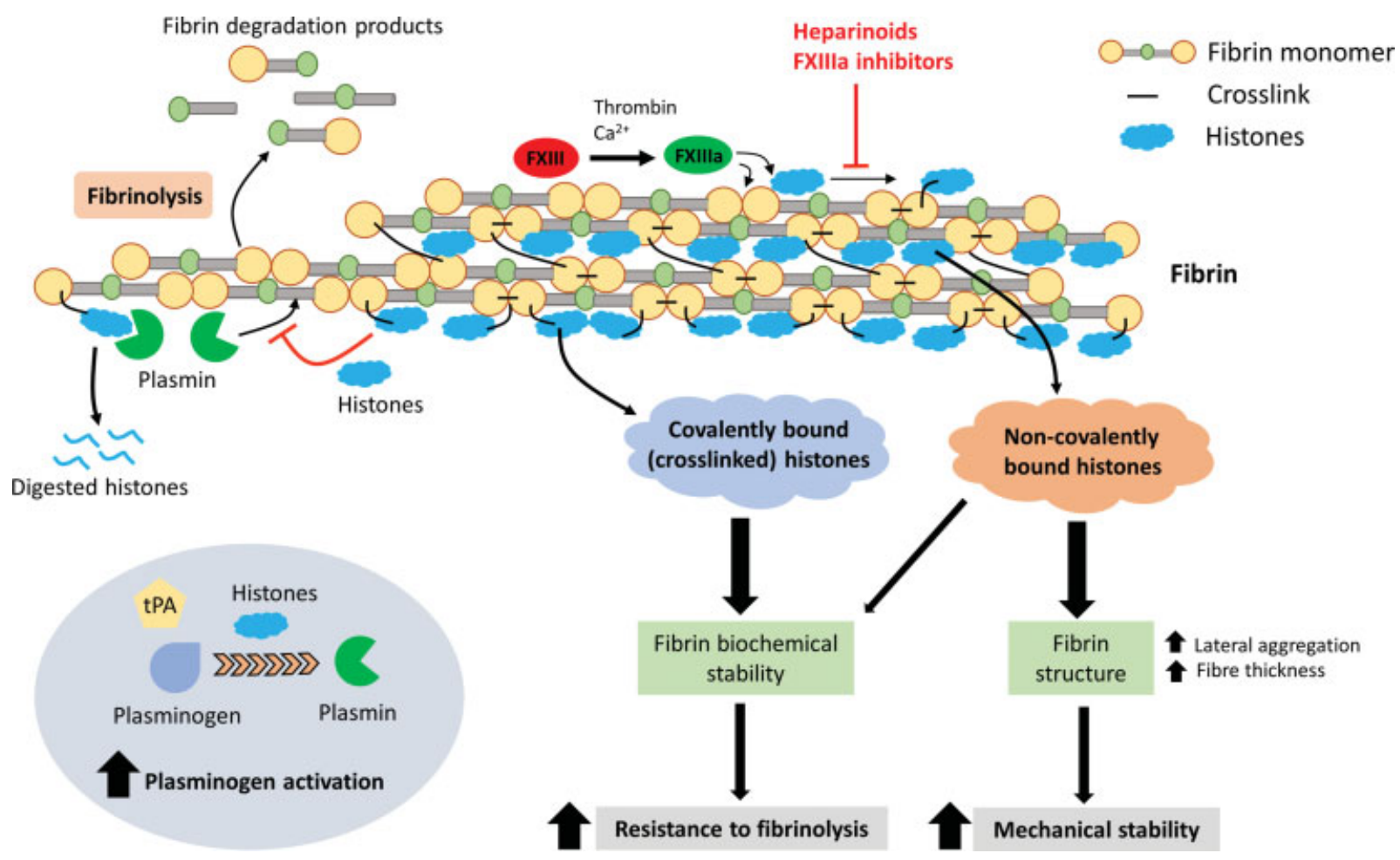

Fig. 6 Summary of histone effects on fibrin stability and fibrinolysis. Histones are associated with fibrin through noncovalent interactions and covalent crosslinking catalyzed by FXIIla. Noncovalent histone-fibrin interactions increase lateral aggregation of fibrin protofibrils and increase fibrin-fiber thickness, giving the fibrin network increased mechanical stability. Histones stimulate plasminogen activation by tPA in the absence of fibrin, possibly by providing a surface for colocalization of reactants through histone-lysine plasminogen-kringle interactions. However, histones competitively inhibit plasmin to delay fibrinolysis, being digested in the process, and protect fibrin from degradation. Crosslinked histones are more effective at inhibiting fibrinolysis than noncovalently bound histones and increase the biochemical stability of fibrin. Blocking histone-fibrin crosslinking with histone-binding heparinoids, or FXIIla inhibitors, improves lysis of clots containing histones. This suggests targeting the FXIII-histone-fibrin axis could be effective in destabilizing clots containing histones to prevent thrombosis. tPA, tissue typeplasminogen activator.

plasminogen has been previously explored in the context of plasminogen's nonfibrinolytic activities in inflammation and cell migration. All histone subtypes bind plasminogen, with histones $\mathrm{H} 1, \mathrm{H} 3$, and $\mathrm{H} 4$ having $K_{\mathrm{D}}$ values in the nanomolar range. ${ }^{34}$ Analogous to cell surface plasminogen receptors, it is possible that histones provide a surface for colocalization of plasminogen and tPA, which is a poor plasminogen activator in solution. As histone concentration increases, reactants could become separated on different histone molecules, or alternatively, competitive inhibition of plasmin might become a factor, explaining the bell-shaped plasminogen activation profile in -Fig. 11. Importantly, the overall effect of histones on fibrinolysis by tPA is inhibitory, indicating that competitive inhibition of plasmin by histones outweighs any stimulatory effects on plasmin generation.

Although our results have been generated in vitro, they have implications for the pathophysiology and treatment of thrombotic disease. By promoting fibrinolytic resistance, histonefibrin crosslinking protects fibrin from elimination, potentially contributing to a thrombotic tendency and resistance to pharmacological therapies. Histones are usually undetectable in circulation, but levels can rise as high as $230 \mu \mathrm{g} / \mathrm{mL}$, for example following trauma, ${ }^{35}$ in line with the concentration range used in this study. In plasma from sepsis patients, histone $\mathrm{H} 3$ was measured at concentrations up to $60 \mu \mathrm{g} /$
$\mathrm{mL}^{36}$ and total histone levels above $75 \mu \mathrm{g} / \mathrm{mL}$ were associated with poor outcome. ${ }^{37}$ Increased levels of circulating histones are also associated with poor prognosis in stroke, myocardial infarction, and venous thromboembolism, and have been identified in patient thrombi. ${ }^{6,8}$ However, it is unclear how circulating levels of histones in prothrombotic conditions correspond to levels found in patient thrombi.

A major source of extracellular histones are neutrophils, which release NETs in response to diverse infectious and noninfectious stimuli. ${ }^{2}$ However, other cell types release histones through NETosis-independent mechanisms including necrosis and apoptosis. ${ }^{1}$ Caution is required when studying NET components such as histones and DNA in isolation, which may not approximate to their activity in NETs. ${ }^{7}$ Isolated NETs have shown inconsistent ability to enhance thrombin generation in the absence of platelets, even though histones possess in vitro procoagulant activity. ${ }^{7}$ The ability of histones to activate coagulation may depend on whether they are in a free, octameric, or DNA-bound (nucleosome) state. It is also possible that NET-associated granular enzymes, such as elastase and myeloperoxidase, in addition to histone modifications such as proteolysis and citrullination, modify the interactions between NETs and the coagulation and fibrinolytic systems. Citrullinated histones are often used as "NET-specific" markers, and citrullinated histone $\mathrm{H} 3$ has been identified in arterial, venous, and 
microvascular thrombi in humans and animals. ${ }^{6,8}$ Although histone citrullination weakens the interaction with fibrinogen, ${ }^{15}$ our results show that it does not prevent binding or FXIIla-mediated crosslinking of histone $\mathrm{H} 3$ to fibrin (-Supplementary Fig. $\mathbf{S 3}$ [online only]).

Histones and DNA exert antifibrinolytic effects, either alone or in combination, ${ }^{11,17}$ but the extent to which histones remain bound to DNA in circulation is unclear. Endogenous DNases promote the degradation of NETs, which would release histones, and their inadequate clearance is associated with thrombosis in animal models. ${ }^{38}$ The presence of DNA and histones in patient thrombi is clinically relevant to clot stability, as the ex vivo lysis of clots retrieved from stroke patients is improved with a combination of DNase and tPA. ${ }^{39}$ However, DNase is not always effective as it only removes the DNA component of NETs, leaving behind histones which mediate tissue injury, initiate thrombosis, and stabilize clots. ${ }^{40-42}$ Our results emphasize that although fibrin is the primary target of thrombolytic therapy, other factors need to be considered for effective fibrinolysis. Fibrin formation and lysis are influenced by numerous fibrin (ogen)-binding proteins and polymers in addition to histones ${ }^{17,24}$ and DNA,, 11 such as Von Willebrand Factor ${ }^{43}$ and polyphosphate. ${ }^{12}$ It is likely that fibrin exists in a complex and heterogeneous network in vivo providing multiple targets for novel therapies.

Targeting FXIIla to prevent thrombosis has a potential advantage over other anticoagulants in that thrombin generation and fibrin formation can still take place, allowing normal hemostasis but reducing potentially thrombotic fibrin networks. ${ }^{23}$ Recently developed small-molecule inhibitors of FXIIla have shown promise in animal models, reducing clot weight without prolonging bleeding in models of venous stasis. ${ }^{44}$ Our results show that histones can be added to the list of FXIIla substrates that inhibit fibrinolysis, which includes $\alpha_{2}$-antiplasmin, TAFI, and PAI-2. ${ }^{19}$ In these cases, crosslinking is proposed to localize antifibrinolytic activity to the fibrin surface and contribute to fibrin stability, and we find that crosslinked histones have a similar effect. It is possible that blocking histone-fibrin crosslinking is a potential antithrombotic benefit of FXIIIa inhibition, which would be relevant for thrombotic disease characterized by histone release.

Heparinoids are the mainstay of anticoagulant treatment and suppress thrombus formation by inhibiting thrombin generation. Histones bind negatively charged heparin and its derivatives with high affinity, ${ }^{32}$ which may be of therapeutic benefit. Heparinoids neutralize the damaging effects of histones in a range of settings, including toxicity in sepsis, ${ }^{45,46}$ thrombosis, ${ }^{5}$ thrombocytopenia, ${ }^{47}$ and platelet activation. ${ }^{48}$ Heparinoids also bind and dismantle NETs, possibly by displacing DNA. ${ }^{5,17}$ LMWH accelerated lysis of clots containing histones (-Fig. 5B-D), which correlated with reduced histone-fibrin crosslinking and increased levels of noncovalently bound histones ( - Fig. 5A). This indicates that LMWH does not prevent binding of histones to fibrin, but rather the crosslinking reaction itself. Increased amounts of noncovalently bound histones were also observed when histone-fibrin crosslinking was prevented by FXIIIa inhibition (-Fig. 2). Presumably under these conditions, histones are bound to fibrin at their crosslinking sites but not covalently attached. However, unlike the situation with FXIIIa inhibition (-Fig. 3), noncovalently bound histones did not increase fibrin clot turbidity in the presence of LMWH (-Fig. 5). The relationship between histones, LMWH, and clot structure is likely to be complex, as LMWH can directly alter fibrin structure, increase clot strength, and delay fibrinolysis. ${ }^{49}$ In our system, LMWH increased clot turbidity, which was reversed by histones (-Fig. 5E), suggesting that LMWH and histones neutralize each other's effects on fibrin structure. Profibrinolytic activities have previously been described for heparin and its derivatives in plasma systems, mainly through reduced TAFI activation, which usually attenuates fibrinolysis. ${ }^{50}$ Preventing histone-fibrin crosslinking and destabilizing clots containing histones are potentially novel antithrombotic and profibrinolytic mechanisms for LMWH beyond anticoagulation, which warrant further investigation.

\section{What is known about this topic?}

- Histones and DNA in the form of neutrophil extracellular traps (NETs) are released into the circulation as part of the innate immune response.

- While beneficial in the fight against infection, NETs contribute to thrombosis by promoting coagulation and stabilizing clots, making thrombi resistant to pharmacological thrombolysis.

- Histones enhance the mechanical and lytic stability of fibrin.

\section{What does this paper add?}

- Histones inhibit plasmin as competitive substrates.

- Histones are noncovalently and covalently associated with fibrin in purified, plasma, and whole blood model systems.

- Covalent crosslinking of histones to fibrin, catalyzed by activated transglutaminase FXIIIa, enhances the ability of histones to inhibit fibrinolysis.

- FXIIIa blockers or histone-binding heparinoids inhibit histone-fibrin crosslinking and improve fibrinolysis, suggesting that the FXIII-histone-fibrin axis can be targeted to destabilize clots to prevent the formation of potentially thrombotic fibrin networks.

Conflict of Interest

None declared.

\section{References}

1 Chen R, Kang R, Fan XG, Tang D. Release and activity of histone in diseases. Cell Death Dis 2014;5:e1370

2 Brinkmann V, Reichard U, Goosmann C, et al. Neutrophil extracellular traps kill bacteria. Science 2004;303(5663):1532-1535

3 Engelmann B, Massberg S. Thrombosis as an intravascular effector of innate immunity. Nat Rev Immunol 2013;13(01):34-45 
4 Gould TJ, Lysov Z, Liaw PC. Extracellular DNA and histones: double-edged swords in immunothrombosis. J Thromb Haemost 2015;13(Suppl 1):S82-S91

5 Fuchs TA, Brill A, Duerschmied D, et al. Extracellular DNA traps promote thrombosis. Proc Natl Acad Sci U S A 2010;107(36): 15880-15885

6 Thålin C, Hisada Y, Lundström S, Mackman N, Wallén H. Neutrophil extracellular traps: villains and targets in arterial, venous, and cancer-associated thrombosis. Arterioscler Thromb Vasc Biol 2019;39(09):1724-1738

7 Noubouossie DF, Reeves BN, Strahl BD, Key NS. Neutrophils: back in the thrombosis spotlight. Blood 2019;133(20):2186-2197

8 Laridan E, Martinod K, De Meyer SF. Neutrophil extracellular traps in arterial and venous thrombosis. Semin Thromb Hemost 2019; 45(01):86-93

9 Wolberg AS. Thrombin generation and fibrin clot structure. Blood Rev 2007;21(03):131-142

10 Longstaff C, Thelwell C, Williams SC, Silva MM, Szabó L, Kolev K. The interplay between tissue plasminogen activator domains and fibrin structures in the regulation of fibrinolysis: kinetic and microscopic studies. Blood 2011;117(02):661-668

11 Gould TJ, Vu TT, Stafford AR, et al. Cell-free DNA modulates clot structure and impairs fibrinolysis in sepsis. Arterioscler Thromb Vasc Biol 2015;35(12):2544-2553

12 Mutch NJ, Engel R, Uitte de Willige S, Philippou H, Ariëns RA. Polyphosphate modifies the fibrin network and down-regulates fibrinolysis by attenuating binding of tPA and plasminogen to fibrin. Blood 2010;115(19):3980-3988

13 Gonias SL, Pasqua JJ, Greenberg C, Pizzo SV. Precipitation of fibrinogen, fibrinogen degradation products and fibrin monomer by histone H3. Thromb Res 1985;39(01):97-116

14 Pemberton AD, Brown JK, Inglis NF. Proteomic identification of interactions between histones and plasma proteins: implications for cytoprotection. Proteomics 2010;10(07):1484-1493

15 Locke M, Francis RJ, Tsaousi E, Longstaff C. Fibrinogen protects neutrophils from the cytotoxic effects of histones and delays neutrophil extracellular trap formation induced by ionomycin. Sci Rep 2020;10(01):11694

16 Kppeć M, Wegrzynowicz Z, Zajdel M, Sawecka J, Szumiel I. Effects of histones and dextran on some properties of fibrin, particularly on its susceptibility to plasmin. Thromb Res 1974;5(03):359-374

17 Longstaff C, Varjú I, Sótonyi P, et al. Mechanical stability and fibrinolytic resistance of clots containing fibrin, DNA, and histones. J Biol Chem 2013;288(10):6946-6956

18 Kalathottukaren MT, Abraham L, Kapopara PR, et al. Alteration of blood clotting and lung damage by protamine are avoided using the heparin and polyphosphate inhibitor UHRA. Blood 2017;129 (10):1368-1379

19 Muszbek L, Bereczky Z, Bagoly Z, Komáromi I, Katona É. Factor XIII: a coagulation factor with multiple plasmatic and cellular functions. Physiol Rev 2011;91(03):931-972

20 Byrnes JR, Duval C, Wang Y, et al. Factor XIIla-dependent retention of red blood cells in clots is mediated by fibrin $\alpha$-chain crosslinking. Blood 2015;126(16):1940-1948

21 Mutch NJ, Koikkalainen JS, Fraser SR, et al. Model thrombi formed under flow reveal the role of factor XIII-mediated cross-linking in resistance to fibrinolysis. J Thromb Haemost 2010;8(09): 2017-2024

22 Rijken DC, Abdul S, Malfliet JJ, Leebeek FW, Uitte de Willige S. Compaction of fibrin clots reveals the antifibrinolytic effect of factor XIII. J Thromb Haemost 2016;14(07):1453-1461

23 Wolberg AS. Fibrinogen and factor XIII: newly recognized roles in venous thrombus formation and composition. Curr Opin Hematol 2018;25(05):358-364

24 Varjú I, Longstaff C, Szabó L, et al. DNA, histones and neutrophil extracellular traps exert anti-fibrinolytic effects in a plasma environment. Thromb Haemost 2015;113(06):1289-1298
25 Longstaff Csubcommittee on fibrinolysis. Development of Shiny app tools to simplify and standardize the analysis of hemostasis assay data: communication from the SSC of the ISTH. J Thromb Haemost 2017;15(05):1044-1046

26 Pieters M, Philippou H, Undas A, de Lange Z, Rijken DC, Mutch NJSubcommittee on Factor XIII and Fibrinogen, and the Subcommittee on Fibrinolysis. An international study on the feasibility of a standardized combined plasma clot turbidity and lysis assay: communication from the SSC of the ISTH. J Thromb Haemost 2018;16(05):1007-1012

27 Carr ME Jr, Hermans J. Size and density of fibrin fibers from turbidity. Macromolecules 1978;11(01):46-50

28 Yeromonahos C, Polack B, Caton F. Nanostructure of the fibrin clot. Biophys J 2010;99(07):2018-2027

29 Herren T, Burke TA, Das R, Plow EF. Identification of histone H2B as a regulated plasminogen receptor. Biochemistry 2006;45(31): 9463-9474

30 Hethershaw EL, Cilia La Corte AL, Duval C, et al. The effect of blood coagulation factor XIII on fibrin clot structure and fibrinolysis. J Thromb Haemost 2014;12(02):197-205

31 Ryan EA, Mockros LF, Weisel JW, Lorand L. Structural origins of fibrin clot rheology. Biophys J 1999;77(05):2813-2826

32 Longstaff C, Hogwood J, Gray E, et al. Neutralisation of the anticoagulant effects of heparin by histones in blood plasma and purified systems. Thromb Haemost 2016;115(03):591-599

33 Köhler S, Schmid F, Settanni G. The internal dynamics of fibrinogen and its implications for coagulation and adsorption. PLOS Comput Biol 2015;11(09):e1004346

34 Nitzsche R, Köhler J, Kreikemeyer B, Oehmcke-Hecht S. Streptococcus pyogenes escapes killing from extracellular histones through plasminogen binding and activation by streptokinase. J Innate Immun 2016;8(06):589-600

35 Abrams ST, Zhang N, Manson J, et al. Circulating histones are mediators of trauma-associated lung injury. Am J Respir Crit Care Med 2013;187(02):160-169

36 García-Giménez JL, Romá-Mateo C, Carbonell N, et al. A new mass spectrometry-based method for the quantification of histones in plasma from septic shock patients. Sci Rep 2017;7(01):10643

37 Alhamdi Y, Abrams ST, Cheng Z, et al. Circulating histones are major mediators of cardiac injury in patients with sepsis. Crit Care Med 2015;43(10):2094-2103

38 Jiménez-Alcázar M, Napirei M, Panda R, et al. Impaired DNase1mediated degradation of neutrophil extracellular traps is associated with acute thrombotic microangiopathies. J Thromb Haemost 2015;13(05):732-742

39 Laridan E, Denorme F, Desender L, et al. Neutrophil extracellular traps in ischemic stroke thrombi. Ann Neurol 2017;82(02): 223-232

40 Kolaczkowska E, Jenne CN, Surewaard BG, et al. Molecular mechanisms of NET formation and degradation revealed by intravital imaging in the liver vasculature. Nat Commun 2015;6:6673

41 Albadawi H, Oklu R, Raacke Malley RE, et al. Effect of DNase I treatment and neutrophil depletion on acute limb ischemiareperfusion injury in mice. J Vasc Surg 2016;64(02):484-493

42 Brill A, Fuchs TA, Savchenko AS, et al. Neutrophil extracellular traps promote deep vein thrombosis in mice. J Thromb Haemost 2012;10(01):136-144

43 Tanka-Salamon A, Kolev K, Machovich R, Komorowicz E. Proteolytic resistance conferred to fibrinogen by von Willebrand factor. Thromb Haemost 2010;103(02):291-298

44 Pasternack R, Büchold C, Jähnig R, et al. Novel inhibitor ZED3197 as potential drug candidate in anticoagulation targeting coagulation FXIIIa (F13a). J Thromb Haemost 2020;18(01):191-200

45 Wang F, Zhang N, Li B, et al. Heparin defends against the toxicity of circulating histones in sepsis. Front Biosci 2015;20:1259-1270

46 Wildhagen KC, Wiewel MA, Schultz MJ, et al. Extracellular histone H3 levels are inversely correlated with antithrombin levels and 
platelet counts and are associated with mortality in sepsis patients. Thromb Res 2015;136(03):542-547

47 Fuchs TA, Bhandari AA, Wagner DD. Histones induce rapid and profound thrombocytopenia in mice. Blood 2011;118(13):3708-3714

48 Semeraro F, Ammollo CT, Morrissey JH, et al. Extracellular histones promote thrombin generation through platelet-dependent mechanisms: involvement of platelet TLR2 and TLR4. Blood 2011; 118(07):1952-1961
49 Komorowicz E, Balázs N, Tanka-Salamon A, et al. Biorelevant polyanions stabilize fibrin against mechanical and proteolytic decomposition: effects of polymer size and electric charge. J Mech Behav Biomed Mater 2020;102:103459

50 Ammollo CT, Semeraro N, Carratù MR, Colucci M, Semeraro F. Histones differentially modulate the anticoagulant and profibrinolytic activities of heparin, heparin derivatives, and dabigatran. J Pharmacol Exp Ther 2016;356(02):305-313 